\title{
Reduced-complexity estimation for Poisson processes modulated by nearly completely decomposable Markov chains
}

\author{
Vikram Krishnamurthy \\ ARC Special Research Centre for \\ Ultra-Broadband Information Networks \\ Department of Electrical \& Electronic \\ Engineering \\ University of Melbourne \\ Parkville, Victoria 3010 Australia \\ e-mail: vikram@ee.mu.oz.au
}

\begin{abstract}
In this paper, we address the problem of complexity reduction in state estimation of Poisson processes modulated by continuous-time nearly completely decomposable Markov chains.
\end{abstract}

\section{INTRODUCTION}

Nearly completely decomposable Markov chains (NCDMC) are usually large scale, and show strong interactions within groups (called "superstates") and weak interactions between the groups. The problem of reduced-complexity estimation for partially observed discretetime NCDMCs was first addressed in [1]. It was shown that for sufficiently small $\epsilon$, (where $\epsilon$ is the parameter that signifies the weak coupling between the superstates) one can obtain $O\left(\epsilon^{2}\right)$ (and under some special cases, $\left.O\left(\epsilon^{3}\right)\right)$ approximations to the aggregate as well as the full-order conditional probability estimates. The number of computations per unit time for this algorithm is $O\left(M^{2}\right)$ instead of $O\left(S^{2}\right)$ where $S$ is the total number of states and $M$ is the number of "superstates" within which the NCDMC shows strong interactions. Typically $M \ll S$, which implies the significant computational savings achievable through this algorithm. Following similar ideas, we address the problem of reduced-complexity state estimation of Poisson processes modulated by continuous-time NCDMCs. Applications of such processes have been identified in studying Markov modulated traffic in queueing networks with bursty traffic (e.g, ATM networks) [2], due to 1) inherent multiple-scale nature of specialized traffic like variable bit rate video traffic and 2) multiplexing of heterogeneous traffic, featuring sources with very different time constants.

\section{State Estimation}

Consider a probability space $(\Omega, \mathcal{F}, P)$. Let $X_{t}, t \geq 0$ be a continuous-time Markov chain defined on this space with state space $\left\{e_{1}, e_{2}, \ldots, e_{S}\right\}$ where $e_{i} \in \mathbb{R}^{S}$ is the unit vector with 1 in the $i$-th position. Let the infinitesimal generator or transition rate matrix be denoted by $A$ where $\sum_{j=1}^{S} a_{i j}=0, \forall i \in\{1,2, \ldots, S\}$. Define $P\left(X_{t}=i\right)=p_{t}^{i}, i \in\{1,2, \ldots, S\}$. The probability distribution $p_{t}=\left(p_{t}^{1} p_{t}^{2} \ldots p_{t}^{S}\right)^{\prime}$ satisfies the forward equation $\frac{d p_{t}}{d t}=A^{\prime} p_{t}$ where $/$ denotes the transpose operation. The Markov chain $X_{t}$ modulates an $L$-variate integrable Poisson process $N_{t}=\left(N_{t}^{(1)} N_{t}^{(2)} \ldots N_{t}^{(L)}\right)^{\prime}$ as follows:

$$
d N_{t}^{(l)}=\left\langle X_{t}, g^{(l)}\right\rangle d t+d m_{t}^{(l)}, l=1,2, \ldots, L
$$

where $N_{t}^{(l)}$ denotes the number of events (e.g, in the case of teletraffic, number of packets arrivals) with mark $l$ that occur during the interval $[0, t]$, and $g^{(l)}=\left(g_{1}^{(l)} g_{2}^{(l)} \ldots g_{S}^{(l)}\right)^{\prime}$ is the vector of intensities of the $l$-th component of the process $N_{t} .\langle\cdot, \cdot\rangle$ denotes the scalar product in $\mathbb{R}^{S}$ and $m_{t}^{(l)}$ is a $\mathcal{F}_{t}$ martingale where $\mathcal{F}_{t}$ denotes $\sigma\left(X_{s}, N_{s} ; s \leq t\right)$. Denote the observation history as $\mathcal{N}_{t}^{(l)}=$

\author{
Subhrakanti Dey \\ ARC Special Research Centre for \\ Ultra-Broadband Information Networks \\ Department of Electrical \& Electronic \\ Engineering \\ University of Melbourne \\ Parkville, Victoria 3010 Australia \\ e-mail: sdey@ee.mu.oz.au
}

$\sigma\left(N_{s}^{(l)}: s \leq t\right)$ and $\mathcal{N}_{t}=\bigvee_{l=1}^{L} \mathcal{N}_{t}^{(l)}$. Note also that for $L=1$, we drop the superscript $l$ in the appropriate notations. Defining an unnormalized measure of $E\left[X_{t} \mid \mathcal{N}_{t}\right]$ as $q_{t}$, we have the following Zakai filter

$$
d q_{t}=A^{\prime} q_{t} d t+\sum_{l=1}^{L}\left(B^{(l)}-I\right) q_{t} d n_{t}^{(l)}
$$

where $B^{(l)}=\operatorname{diag}\left[g^{(l)}\right]$ and $n_{t}^{(l)}=N_{t}^{(l)}-t$.

Considering the univariate case $(L=1)$, a robust time-discretized approximation to $q_{t}$ (by sampling at regular time intervals separated by $h$ ), can be written as (following similar techniques as in [3])

$$
\alpha_{n+1}=\alpha_{n}(I+A h) C_{n+1}
$$

where $\alpha_{n}=q_{n}^{\prime}$ and $C_{n+1}$ is a diagonal matrix with the $i$-th diagonal entry being $\exp \left[-\left(g_{i}-1\right) h\right] g_{i}^{\Delta N_{(n+1)}}, \triangle N_{(n+1)}=N_{(n+1) h}-N_{n h}$.

Note that $(I+A h)$ is a stochastic matrix and the normalized conditional probability estimate $\hat{\alpha}_{n}$ can be obtained as $\hat{\alpha}_{n}=\frac{\alpha_{n}}{\left\langle\alpha_{n}, \underline{1}\right\rangle}$ where $\underline{1} \in \mathbb{R}^{S}$ is a column vector of all 1 's.

\section{REDUCED-COMPLEXITY ESTIMATION WITH NEARLY COMPLETELY DECOMPOSABLE MARKOV CHAINS}

For a nearly completely decomposable structure, the transition probability matrix of the Markov chain is given as $\bar{A}+\epsilon \bar{B}$ where $\bar{A}$ has a block diagonal structure where the $i$-th block $\bar{A}_{i i} \in \mathbb{R}^{s_{i} \times s_{i}}, \forall i$, $\sum_{i} s_{i}=S, \epsilon>0$ is a small perturbation parameter, and $B \in$ $\mathbb{R}_{i}^{S \times S} . \bar{A}_{i i}, \forall i$ are also infinitesimal generators and we assume that $A$ and $\bar{A}_{i i}, \forall i$ are irreducible and aperiodic. We also assume that the intensities of the Poisson arrival process only depend on the state partitions, i.e, $g_{i}=\bar{g}_{j}, \forall X_{t}=e_{i} \in \mathcal{S}_{j}$. Using the same decoupling techniques as in [1], it can then be shown that when the above assumptions hold and $\epsilon$ is sufficiently small, one can obtain $O\left(\epsilon^{2}\right)$ approximations to $\alpha_{n}, \hat{\alpha}_{n}, \forall n$ with $O\left(M^{2}\right)$ computations rather than $O\left(S^{2}\right)$ computations as demanded by (3). Since typically $M \ll S$, this implies a substantial reduction in computations. For all related details, see [1].

\section{REFERENCES}

[1] S. Dey, "Reduced-complexity filtering for partially observed nearly completely decomposable Markov chains," IEEE Transactions on Signal Processing, December 2000. to appear.

[2] K. Kontovasilis and N. Mitrou, "Markov-modulated traffic with nearly complete decomposability characteristics and associated fluid queueing models," Advanced Applied Probability, vol. 27, pp. 1144-1185, 1995.

[3] J. Clark, "The design of robust approximations to the stochastic differential equations for nonlinear filtering," in NATO Advanced Study Institute, Series E, Applied Sciences, vol. 25, pp. 721-734, Alphen Aan Den Rijn: Sijthoff and Noordhoff, 1978. 